\title{
Tratados de Libre Comercio y mercados regionales de trabajo. MERCOSUR y la agricultura tradicional en Chile ${ }^{1}$
}

\author{
Antonio Daher
}

Los balances agregados de empleo en la evaluación de los tratados comerciales ocultan desbalances sectoriales y regionales, como sucederá por efecto del MERCosUR en la agricultura tradicional y en las regiones del centro-sur de Chile, donde se reducirán entre 50 y 70 mil empleos directos. Este impacto laboral en cuatro regiones contiguas (VII, VIII, IX y X), que representan el $43 \%$ del producto silvoagropecuario y el $55 \%$ de la fuerza de trabajo del sector, agudizará críticamente la pobreza e indigencia rurales, radicadas en más de dos tercios en dichas áreas. A la reducción cuantitativa de empleos debe agregarse un cambio cualitativo en la estructura de categorias ocupacionales relacionado con la reconversión de la agricultura tradicional campesina en otra moderna y empresarial. Ello incidirá en los mercados regionales de trabajo, definidos -en la agricultura chilena- no sólo territorialmente, sino también por una especialización subsectorial, categorias ocupacionales y relaciones laborales especificas, y un ámbito social y cultural propio. Las opciones y restricciones a la reconversión agricola y los ajustes laborales y demográficos -inchuidas migracionescuyos costos económicos y sociales se desconocen, reclaman respuestas sectoriales y politicas regionales aún pendientes.

\section{Introducción.}

Los probables efectos macroeconómicos, sectoriales y territoriales de los Tratados de Libre Comercio (TLC) en Chile impactarian diferencialmente

\footnotetext{
${ }^{1}$ Ponencia ante el "III Seminario Internacional Impactos Territoriales de los Procesos de Reestructuración", Universidad Internacional de Andalucia, Sede Iberoamericana, La Rábida, Espafta. El autor, académico de la Pontificia Universidad Catolica de Chile, agradece el patrocinio de la Dirección de Investigación y Postgrado de la misma Universidad.
} 
los mercados de trabajo regionales. ${ }^{2}$ Una evaluación de tales efectos permite anticipar que sin bien a nivel del empleo agregado el balance sería equilibrado y hasta positivo, sectorial y regionalmente se producirían desequilibrios significativos. Estos últimos se acusarían de manera especialmente crítica en el subsector de agricultura tradicional sustitutiva de importaciones, concentrado fuertemente en las regiones del centro-sur y sur (regiones VII a X), en cuyas áreas rurales se encuentra, además, la mayor pobreza relativa del país. Si bien se prevé cierta opción de reconversión, algunas rigideces estructurales inhabilitarian tal posibilidad o la harian altamente costosa en términos económicos y sociales.

Los Tratados de Libre Comercio, aunque puede presumirse que, en términos generales, resultan beneficiosos para la economía y la sociedad de los países que los suscriben, también pueden afectar muy diferencialmente a los sectores y subsectores como asimismo a los territorios, a las regiones y espacios subregionales.

Como se ha verificado en estudios recientes relativos a la asociación de Chile al MERCOSUR y a su eventual incorporación al NAFTA (Daher 1996 a y b; Hachette y Morales, 1996), determinados sectores económicos resultan más beneficiados que otros, y algunos subsectores definitivamente pierden. La traducción territorial de estos efectos de distinto signo identifica a su vez regiones perdedoras y ganadoras con cada TLC (o con una particular combinación de éstos que, simultánea o secuencialmente, pueden detonar un impacto agregado que magnifique las polaridades, o que las minimice o anule cuando los efectos son opuestos).

Se prefigura así un "seccionamiento geográfico", una "fuerte y odiosa división geográfica" con graves implicancias económicas y sociales (Gazmuri, 1996). En extremo ello agudizaría la concentración, creciendo por ejemplo el PIB (Producto Interno Bruto) de la Región Metropolitana en $6 \%$ por efecto del MERCOSUR, mientras que el PIB de la IX Región caería en un porcentaje similar (García Ruminot, El Mercurio, 27 de julio de 1996).

\footnotetext{
${ }^{2}$ Chile está dividido administrativamente en 12 regiones, enumeradas correlativamente de norte a sur, más une Región Metropolitana localizada en el centro del país (véase mapa adjunto al final del texto). La población esta mayoritariamente distribuida entre las regiones IV y X, con una fuerte concentración en ia zona central -regiones Metropolitana y V-y en la VIII Región del Bio-Bío. El producto económico presenta una concentración análoga en estas ủltimas regiones, destacando además la II Región de Antofagasta, la principal en gran minería. Sectorialmente las regiones I y $\mathrm{XI}$ son predominantemente minero-pesqueras; la II es minera; las regiones III, IV y VI son minero-agricolas; la VII es agro-energética; la IX y X son silvoagropecuarias y, esta última, también pesquera; la XII Región es minero-energética; y las regiones Metropolitana, V y VIII tienen economias más diversificadas basadas un recursos naturales, industria y servicios.
} 


\section{A. Daher / Tratados de Libre Comercio y mercados ...}

Detrás de estos impactos diferenciales está ciertamente la disímil geografia física, económica y social de cada país, y más específicamente la desigual competitividad internacional de los sectores y regiones, como también su diversa sensibilidad frente a uno u otro tratado (Escobar y Repetto, 1993 ; Daher, 1996 b).

Los Tratados de Libre Comercio afectan heterogéneamente la estructura productiva, el comercio exterior y las inversiones en cada región, según sea su competitividad expresada en el grado de apertura y transabilidad de la economia regional, su especialización sectorial, el destino geográfico de sus exportaciones y el mix de países competitivos de su sector sustituidor de importaciones, y ciertamente también según sean sus ventajas urbanas y de infraestructura.

La inserción de Chile en los mercados internacionales expresada en el incremento y la diversificación de sus exportaciones, ${ }^{3}$ encuentra su origen en las reformas estructurales que detonaron la liberalización y la apertura económica, explicando a su vez los significativos ajustes sectoriales y territoriales ocurridos en Chile en los dos últimos decenios. Tales cambios predisponen a las economías regionales ante los efectos previsibles de la asociación del pais a ciertos Tratados de Libre Comercio, y permiten prefigurar los nuevos ajustes y reconversiones territoriales inducidos por estos (Daher, $1996 \mathrm{a})$.

Los efectos probables de los TLC han detonado una fuerte polémica pública, con opiniones polarizadas tanto en el ámbito político como en el gremial. Lo cierto es que, firmada recientemente la asociación de Chile al MERCOSUR, parece haber unanimidad -o al menos una amplia convergencia-en relación a que, más allá de los beneficios globales esperados, e incluso aceptando un probable saldo neto positivo para el sector agrícola en su conjunto, la agricultura tradicional, vinculada principalmente a los cultivos anuales y más especificamente a los cereales, acusará un importante impacto negativo.

En el medio académico, estudios especializados lo han cuantificado con relativa precisión, tanto a nivel de tipo de cultivos, como de super-

\footnotetext{
${ }^{3}$ Las exportaciones chilenas crecen de menos de US\$500 miliones en 1960 a más de US\$11.500 en 1994. En moneda de este ültimo año, este valor es 6,5 veces superior al de 1960, y 2,6 veces mayor al de 1985. Desde 1974 en adelantc, la expansión anual de las exportaciones supera a la del producto, y entre 1985-1994 las exportaciones crecen a un promedio anual de $10,3 \%$ mientras el producto lo hace a un promedio de $6,2 \%$. La participación relativa de las exportaciones en el producto (a precios constantes de 1986) pasa de cerca del 12\% entre 1960-75 a un 35\%, aproximadamente, en los noventa. Este crecimiento involucra una imporlante diversificación sectorial: en iguales períodos, la participación del cobre en la canasta exportadora cae de más del $70 \%$ a menos del $40 \%$, destacando en 1994 el aporte de los productos forestales (13,9\%), del mar (11,4\%), agricolas $(9,4 \%)$, mineros no-cobre $(8,1 \%)$ y otros $(20,7 \%)$ (Melicr y Sáez, 1995).
} 
ficies y niveles de producción afectados, además de identificar las regiones mayormente involucradas (Quiroz, Larraín y Labán, 1995; Errázuriz y Muchnik, 1996; Muchnik, Errázuriz y Domínguez, 1996).

El sector agrícola es, según todos los análisis, -el más afectado, potencialmente, por ambos tratados. Su despliegue territorial en un conjunto significativo de regiones, su carácter extensivo en contraste con los otros sectores, la relación que establece con un sinnúmero de asentamientos menores y medianos $y$, en fin, su significativa relevancia en términos de empleo y su asociación, muchas veces con focos de pobreza, determinan que dicho sector sea aquí más pormenorizadamente considerado en relación a los objetivos de esta investigación.

Sin embargo, hasta el momento ningún estudio ha sido hecho con el propósito de cuantificar el costo del ajuste y de la reconversión sugerida. Esta última, por lo demás, apenas ha sido insinuada en términos técnicos, y su propia factibilidad -según las calidades de suelo, climas, fraccionamiento predial, ciclos de rotación, capacitación del pequeño empresariado y calificación de la mano de obra, acceso al crédito, etc.- no ha sido aún demostrada. El ajuste y la reconversión -dilatados por los mayores plazos de desgravación negociados (que alcanzan como máximo a 18 años)- no sólo involucrarán un costo económico probablemente muy alto, sino también un costo social de magnitud considerable.

Este estudio apunta precisamente a una primera aproximación de medición del impacto laboral del MERCOSUR en la agricultura tradicional chilena, relacionándolo con el condicionamiento territorial de los mercados de trabajo y los procesos de movilidad geográfica y sectorial de ese factor, y los consiguientes flujos migratorios que exceden a la sola fuerza de trabajo.

En el primer capitulo se hace una muy breve reflexión conceptual relacionando el comercio internacional con los mercados nacionales y subnacionales de factores productivos, introduciendo así al análisis más pormenorizado que, en el segundo capítulo, se dedica a los efectos de los TLC, y en particular del MERCOSUR, en la agricultura y las regiones chilenas. El tercer capítulo está referido a los mercados regionales de trabajo, con especial énfasis en el impacto de MERCOSUR en el empleo agrícola, y luego a las opciones y restricciones que enfrentará la reconversión del sector. Finalmente, se relatan las principales conclusiones relacionadas con los temas descritos. 


\section{Comercio internacional y mercados subnacionales de factores.}

La dotación diferencial de factores productivos, como base del comercio internacional, y la tendencia a la convergencia en sus precios, como resultado del mismo, connotan a la vez una especificidad sectorial y otra teritorial. Esta última, obviamente constitutiva del problema toda vez que:se trata de paises entre los cuales se produce el intercambio, se manifiesta asimismo intranacionalmente dada la diferencia de factores entre regiones y localidades, y sus respectivos intercambios.

No casualmente, entonces, los tratados y acuerdos de libre comercio, más allá de sus efectos macroeconómicos agregados, producen impactos sectoriales y territoriales diferenciados, afectando los mercados de factores, sus precios y su asignación en el ámbito subnacional.

El efecto de ló Tratados de Libre Comercio sobre los mercados regionales de trabajö sé liga, conceptualmente, a la relación entre comercio y diferencial salarial. La teoría de Heckscher, Ohlin y Samuelson plantea que el patrón de comercio de un pais (o región) está determinado porr la dotación relativa de factores productivos, basánđose el intercambio en las diferencias relativas de los mismos -incluido el trabajo- que póseen los países (o regiones). El propósito del comercio sería reducir los efectos generados por tales diferencias, pudiendo inferirse que el incremento de aquel generaria una tendencia hacia la igualdad o convergencia (internacional o interregional) del precio de los factores, entre ellos el del trabajo. A su.vez, el teorema de Stolper y Samuelson plantea que la existencia de barreras comerciales "ayuda". al factor escaso, aumentando el pago que recibe y, por el contrario, el libe comercio "perjudica" al factor escaso y "beneficia" al factor relativamente más abundante, mediante el cambio de precios relativos de los bienes (Meller y Tockman, 1996):

Esto sucéde porque cuando dos paises (o regiones) comercian entre si, intercambiàn no sólo biencs, sino, implícitamente, también factores productivos incorporados en tales bienes. Sin embargo, dentro de un país, habrá sectores ganadores y sectores perdedores, ya que los cambios ẹn los precios relativos de los bienes (generados por el comercio) alterarán el precio relativo de los factores productivos, afectando así la distribución del ingreso (Meller y Tockman, 1996). Por extensión, según la distribución geográfica de los sectores ganadores y perdedores, y según la proporción diferencial de capital y trabajo entre regiones, habrá también regiones ganadoras y perdedoras, y los cambios en la distribución del 
ingreso implicarán también transferencias interregionales del mismo. Algunas consecuencias de esto se observarán al momento de evaluar los impactos sectoriales y regionales del comercio con MERCOSUR.

La experiencia de intercambio comercial en MERCOSUR plantea, frente a los conceptos anteriores, complementariedades sugerentes no sólo en el plano intersectorial, sino especialmente en el intrasectorial. En efecto, el comercio bilateral entre Argentina y Brasil tiene un componente de bienes manufacturados mayor que en sus respectivas exportaciones al resto del mundo. De hecho, el $45 \%$ de las ventas argentinas al Brasil y el $83 \%$ de las brasileñas a Argentina corresponden a este sector. En cambio, sólo el 25 y el $55 \%$ de las exportaciones al resto del mundo de ambos países, respectivamente, son manufacturas (Ferrer, 1996). Cabe recordar que, para Chile, MERCOSUR representa también un mercado singular, diferenciado claramente del resto del mundo: las ventas chilenas de manufacturas al MERCOSUR representan cerca de un tercio del total de la canasta exportadora a ese mercado, mientras que el mismo sector aporta sólo entre el 1 y el $13 \%$ del total exportado a otros continentes o mercados regionales (Daher, 1996 a).

La constatación anterior podría sugerir una mayor competitividad manufacturera al interior de un mercado subregional con niveles similares de industrialización y tecnología, y a la vez la búsqueda de mercados ampliados, en el ámbito subregional, para una cierta proporción de una industria inicialmente sustitutiva.

Al mismo tiempo, en materia de salarios, se verifica, al menos en Chile y Brasil, que las exportaciones se concentran en los sectores con salarios mayores (Meller y Tokman, 1996; Escobar y Meller, 1996).

Estos últimos autores han demostrado, en la experiencia chilena reciente de apertura comercial (1984-91), que el diferencial de remuneraciones entre las regiones y el Gran Santiago (Región Metropolitana) presenta una evolución opuesta en empresas grandes y pequeñas. En efecto, en las primeras, el salario promedio regional en 1984 año de inicio del auge exportador nacional-alcanzaba sólo al 75\% del correspondiente a la R.M. En 1991, en cambio, dicho salario:superaba en. 12\% al de esta última. Por el contrario, el diferencial salarial entre pequeñas empresas regionales y metropolitanas se acrecentó, en igual período, del $5 \%$ al $12 \%$ en favor de estas últimas (Escobar y Meller, 1996). En el mismo estudio se concluye que el modelo exportador -a diferencia de la anterior estrategia de industrialización sustitutiva de importacioneshabría tenido además un efecto desconcentrador -a favor de las regionesdel producto nacional, destacando sin embargo que ciertas regiones, 
presumiblemente no exportadoras, estarían quedando marginadas del crecimiento económico del país. Esta última conclusión, ciertamente relevante para los mercados regionales de trabajo, es coincidente en cierta medida con la propuesta por Ortiz (1996) para el caso mexicano, donde se afirma que existen alguinos estados tan subdesarrollados que nada tienen que ver con las cifras laborales en la esfera nacional:

Los balances macro de empleo más o menos positivos o equilibrados no necesariamente sustituyen los desbalances sectoriales $y$, sobre todo, territoriales, dadas la imperfecta movilidad del factor trabajo y las dificultades -incluso estructurales-parala recapacitación y reconversión del factor.

La movilidad geográfica del trabajo involucra como es sabido, costos individuales (económicos, psicológicos, etc.), familiares (desarraigo, desvinculación de parejas e hijos) e incluso costos sociales. Entre estos últimos cabe destacar los de las migraciones rural-urbanas, que detonaron una inflación demográfica en las principales ciudades, rodeándolas de cinturones de marginalidad y pobreza, y luego, aquellos inherentes al fenómeno de los temporeros y a la nueva "agrourbanización" para la exportación (Daher, 1987).

Por ende, los mercados de trabajo, si bien presentan ajustes intersectoriales e interregionales -e incluso internacionales-poseen un "área de mercado" que les confiere un marcado carácter local. En cierta medida, se trata de un factor de transabilidad media entre la alta movilidad del capital y la inmovilidad de la tierra y sus recursos.

Amén de los costos ya señalados y los de transportes, contribuyen a esta "cautividad" de mercado rigideces legales -sobre todo en el plano internacional-e incluso sindicales (por rama, empresa, etc.) que, entre otros efectos, limitan el reequilibrio vía salarios.

La evidencia estadística muestra, en consecuencia, persistentes disparidades en las tasas de crecimiento de la fuerza de trabajo, el desempleo y la cesantía entre las distintas regiones.. Es más; la evolución de los salarios confirma, en sus diferencias territoriales amén de sectoriales, el ámbito local de los mercados de trabajo (Mellery Tokman; 1996; Escobar. y Meller, 1996).

La existencia de "regiones ganadoras y regiones perdedoras" (Daher, 1994) en el marco de la apertura e integración económica marcará decisivamente los mercados laborales regionales, y, según se sostendrá más adelante, estos mercados de trabajo no sólo están territorialmente definidos -por su carácter local-sino también por una especialización sectorial y subsectorial $y$, en el caso de la agricultura chilena, por 
relaciones laborales y productivas propias, en un ámbito socioeconómico y cultural especifico.

\section{Las dos agriculturas y el libre comercio: regiones ganadoras y perdedoras.}

La realidad virtual de "dos agriculturas", una empresarial y otra campesina, una moderna y otra tradicional, una exportadora y otra sustitutiva, una del centro y otra del sur, una ganadora y otra perdedora, permite -más allá de la metáfora y su esquematismo-prefigurar los efectos del libre comercio en la agricultura chilena que, como se verá, resultarán complejos y matizados. En la dimensión territorial, la asociación de la agricultura tradicional a un conjunto de regiones geográficamente contiguas validará el posterior análisis -mediante el efecto en el empleo- de mercados regionales de trabajo especificados territorial y sectorialmente, pero sobre todo por singulares relaciones sociales y culturales.

La revisión de los efectos de los tratados -NAFTA y MERCOSUR- y acuerdos de libre comercio -con los Estados Unidos y Argentinapermite verificar no sólo impactos subsectoriales $-\mathrm{y}$ por rubro-de signo opuesto. En el caso del sector agropecuario chileno, y dado el despliegue geográfico de sus componentes, ello se traduce en efectos territoriales -regionales, provinciales y comunales-más o menos positivos o negativos. Esto, a su vez, detona ajustes en los mercados locales de factores -entre ellos los del trabajo-modificando su asignación y precio.

\section{a) Efectos del libre comercio en la agricultura chilena.}

Un primer estudio evaluativo (Muchnik et.al., 1992), realizado sólo para el escenario de un ALC (Acuerdo de Libre Comercio) con Estados Unidos, establece que si se eliminaran por completo los aranceles generales y específicos aplicados en Chile -que fluctuaban entre 14 y $35 \%$ en 1989-1991-, las importaciones nacionales en los rubros agropecuarios podrían incrementarse hasta en un $50 \%$, con un efecto mayor en el caso del trigo seguido, paritariamente, por los correspondientes al maíz, aceite y azúcar. Por otro lado, las exportaciones del sector también se incrementarían, pero sólo en alrededor del $40 \%$ del aumento de las importaciones, aunque, sumadas a las agroindustriales, superarian en más del $20 \%$ a dicho aumento. Este último rubro sería el más beneficiado, puesto que enfrenta mayores niveles de protección arancelaria. En cambio, la fruta fresca -con la sola excepción de la uva, cuyas ventas se ven 
estacionalmente restringidas por una Orden de Comercialización en los Estados Unidos- no verían alteradas sus exportaciones, las cuales no tienen barreras arancelarias o para-arancelarias. (La metodología usada en esta evaluación, según sus autores probablemente sobreestima la contracción de la producción y el aumento de las importaciones, y subestima las exportaciones).

Un estudio más reciente (Quiroz, Larraín y Labán, 1995), realizado esta vez para analizar los efectos del NAFTA y el MERCOSUR en los sectores agricola y agroindustrial, establece que, en términos generales; el $73 \%$ de las importaciones de cereales -el trigo, el maiz y el arroz representan, por sí solos, el $40 \%$ del total de importaciones sectorialesprovienen del bloque NAFTA, y un $25 \%$ del MERCOSUR, de donde se concluye que la suscripción simultánea de ambos acuerdos equivaldría prácticamente al libre comercio total de cereales. En el caso de las exportaciones -donde uva, manzanas, ciruelas, kiwis y peras equivalen al $67 \%$ del total del sector agrícola y agroindustrial-, un $53 \%$ de las ventas totales de fruta se concentra en el NAFTA y sólo un 5\% en el MERCOSUR, ocurriendo algo similar con las exportaciones de hortalizas y semillas, de lo que se deduce que, con pocas excepciones, MERCOSUR resulta ser sistemáticamente de poca importancia para la canasta exportadora agricola y agroindustrial chilena. En contraste, en términos de las importaciones sectoriales nacionales, el $100 \%$ de la carne de vacuno $(27 \% \mathrm{del}$ consumo local en 1993) y de los aceites proviene del MERCOSUR

A largo plazo, las estimaciones econométricas sectoriales llevan a la conclusión de que, inequivocamente, el NAFTA es mejor para la agricultura chilena que el MERCOSUR, ya que éste impone más costos y genera menos beneficios. Con el NAFTA se afectan sólo algunos cultivos tradicionales (trigo, maíz y arroz) pero los demás no se afectan o mejoran parcialmente (remolacha), mientras que los productos exportables en general muestran un efecto positivo. Con MERCOSUR, en cambio, sufren significativamente, además del trigo y el maíz, las oleaginosas, y probablemente (en 1995 aún no se definían los términos del acuerdo), el azúcar y la remolacha. Estos mayores efectos negativos se comparan con menores ganancias en exportaciones, al ser MERCOSUR un mercado menor para ellas (Quiroz, Larraín y Labán, 1995).

El NAFTA representa el $26 \%$ de las exportaciones de cereales y derivados chilenos y -como se ha señalado-el $73 \%$ de las importaciones nacionales en los mismos rubros. Esto es particularmente importante, en términos de competitividad, si se considera que los rendimientos chilenos se sitúan frecuentemente al nivel de las naciones desarrolladas, y que el 
súbsector de cultivos anuales, aún habiendo disminuido en un tercio su súperficiè eñ las últimas ocho temporadas, compromete cerca de un millón de hectáreas y aporta el $30 \%$ del valor total de la prodúcción agropecuaria. Se plantea así la conveniencia de no disminuir el nivel de protección de este subsector mientras no se advierta una reciprocidad por parte del NAFTA, y de negociar acuerdos bilaterales agrícolas análogamente a los celebrados entre los paises de ese Tratado (Véliz, 1995).

Complementariamente, debe señalarse que un ALC con Argentina tendría como principal efecto la transferencia de recursos de algunos productores agrícolas a los consumidores nacionales: los productores de trigo y de azúcar transferirian al menos un 20 y un $66 \%$, respectivamente, de los valores brutos de su producción (Hachette y Larraín, 1994). Esta redistribución de recursos, en lo espacial, tiene un sello de movilidad geográfica, desde la regiones productoras hacia los consumidores en general dispersos aunque mayormente localizados en las grandes áreas metropolitanas. Involucra, pues, una transferencia interregional de recursos.

Además, es necesario tenér presente que las implicancias de la Ronda Uruguay de la Organización Mundial de Comercio (OMC) para el sector agrícola chileno se expresan en una reducción de $41 \%$ (al cabo de 6 años) en el promedio nominal de aranceles pagados por los principales productos agrícolas exportados a sus mayores mercados, y especificamente dé $25 \%$ en el caso de Estados Unidos. La mayor apertura de mercado se produce en Canadá donde los aranceles tenderán a bajar de 76 a 46\%, lo que equivale al $17 \%$ del precio bruto inicial de venta de los bienes (Tironi, 1995).

La balanza comercial sectorial -agropecuaria y agroindustrial-acusará cambios importantes por efecto de la asociación chilena al MERCOSUR. Las exportaciones a ese bloque aumentarán en 15\%, mientras las importaciones provenientes de MERCOSUR crecerán en $109 \%$ como consecuencia tänto de la creación como de la desviación de comercio. La exportaciones totales del sectór sólo se incrementarán en $2 \%$ y las importaciones totales eǹ 19\% (Muchnik, Errázuriz y Domínguez, 1996).

La producción de cereales, según la misma fuente, se veria afectada en los casos del maiz, con una caída del $9 \%$, respecto de 1995 , entre la vigencia del Tratado y el año 2006; del trigo, con una reducción del 16\% entre el 2007 y el 2014; y del arroz, con un decremento del $4 \%$ mayormente concentrado entre el 2006 y el 2011, según los respectivos programas de desgravación. Los índices de producción de pecuarios y cultivos industriales acusarian, a su vez, bajas de $19 \%$ en la carne bovina que se 
estabilizan en el año 2011; del $47 \%$ en la remolacha y de $52 \%$ en las oleaginosas, entre el 2007 y el 2011.

Las caídas de producción mencionadas se traducirían en una disminución potencial de la superficie de cultivos que alcanzaría, concluido el período de desgravación arancelaria, a 106.408 hectáreas, desagregadas en 61.959 hectáreas correspondientes a trigo, 24.873 a remolacha, 9.566 a maíz, 8.591 a oleaginosas y 1.419 a arroz. La pérdida de bienestar de los productores de bienes importables alcanzaría un valor total -valor presente de las pérdidas anuales en los próximos 20 años-de US $\$ 265,8$ millones, correspondiendo 81,1 a carne de bovinos; 67,9 a maíz; 56,0 a trigo; 41,8 a remolacha; 16,3 a arroz y 2,5 a oleaginosas (estas pérdidas contrastan con ganancias totales de US\$ 118,4 millones de los productores de bienes exportables del mismo sector) (Muchnik, Errázuriz y Domínguez, 1996).

\section{b) La agricultura tradicional y las regiones perdedoras.}

Según los mismos autores, la distribución territorial de las pérdidas señaladas -repartidas según la distribución regional de los cultivos durante la temporada 1994/95- afectaría en orden decreciente a las siguientes regiones: VI, con pérdidas de US\$ 57,2 millones; VII, con US\$ 56 millones; VIII, US\$ 45,8 millones; IX, US\$ 39 millones; y X, US $\$ 35,7$ millones. Las regiones Metropolitana y V, ambas de relevancia sectorial, tendrían en conjunto pérdidas sólo de US\$ 18,7 millones, mientras que el resto de las regiones del país acumularia una cifra negativa de US\$13,4 millones.

El conjunto de las cuatro regiones más afectadas y con menos beneficios intrasectoriales compensatorios (VII, VII, LX y X) concentraría, pues, dos tercios de las pérdidas totales del país (66,3\%). Al interior de estas regiones, el origen por rubro de la mayor proporción de pérdidas es el siguiente: en la VII Región, un 35\% de ellas se asocia a la remolacha, y cerca de un $36 \%$ se vincula, en partes iguales, al maiz y al arroz. En la VIII, cerca del $64 \%$ de las pérdidas se produce, en partes equivalentes, por el trigo y la remolacha, y un $25 \%$ por el rubro bovinos. En la $D X$, casi la mitad de la pérdida regional se origina en el trigo, y otro significativo $43 \%$ en los bovinos. Finalmente, en la X Región, más del $80 \%$ de aquélla se deriva de la ganadería bovina (Muchnik, Errázuriz y Domínguez, 1996).

Debe tenerse presente que los beneficios esperados -asociados principalmente a frutas y hortalizas y a su agroindustria, como también a 
vinos y alcoholes-se localizan, mayoritariamente, en porcentajes súperiores al $50 \%$ en las regiones del centro del pás (V, VI y RM), contrarréstando así las respectivas pérdidas, e incluso revirtiêndolas. Esto determina que el área de regiones perdedoras corresponda à las cuatro regiones contiguas del centro-sur (VII y VIII) y sur (IX y X). (La VII Región, si bien detenta porcentajes importantes -aunque nơ los principales- de la producción de pomáceas, kiwis y tomates; no alcanza a revertir sus pérdidas, dado el mayor peso de los importables sobre los exportables).

La distribución regional de las pérdidas, asignadà en proporción a la distribución regional de la superficie de cada cultivo, puede ser corregida y précișada en función de los diferenciales regionales de rendimiento para cada uno de ellos. Asi por ejemplo, el trigo régistra en las regiones Metropolitana y X, en el período 1985-94, rendimientos superiores hasta en un $50 \%$ sobre los correspondientes a las regiones VI a IX, no presentándose diferencias sustantivas entre éstás últimas. En el caso del maíz, los rendimientos mayores se registran en la VI Región, bajando en la Metropolitana y más aún en la VII: en esta última, ellos equivalen al $60 \%$ de los de la VI. Para el arroz, las cifras son equivalentes en las regiones VII y VIII, y superiores en aproximadamente $20 \%$ en la VI. Los rendimientos de maravilla caen en la VIII respecto de la VII, y los de raps suelen ser mayores en la $\mathrm{X}$. La remolacha presenta, a su vez, rendimientos decrecientes de norte a sur, entre la VI y la $X$ regiones (Faiguenbaum, 1996). .

Los datos anteriores indicarían que las regiones con mayores rendimientos sostenidos en determinados cultivos estarian en mejor situación competitiva y que, por ende, el impacto negativo, calculado sólo proporcionalmente a la distribución regional de superficies, sería presumiblemente menor en ellas y mayor en las con rendimientos menores.

En concreto, esto significaría que, en trigo, las regiones VII a IX podrían acusar mayores pérdidas. Otro tanto sucedería con la VII Región en el caso del maiz. Y en el de la remolacha, con las regiones más sureñas (VIII, DX y X).

El impacto negativo sobre algunos subsectores de la agricultura resulta agravado por i) involucrar un área geográfica especifica que compromete a cuatro regiones contiguas (de las trece que integran el país); ii) implicar a una proporción muy alta de la población rural de todo Chile, precisamente radicada en dicha área; iii) afectar a pequeños y medianos propietarios y empresarios agrícolas, a comunidades indígenas y en general a sectores pobres y de extrema pobreza, localizados en esas 
regiones y vinculados a la agricultura tradicional; iv) amenazar formas de vida y culturas arraigadas, en la ruralidad y; en ciertas zonas; a etnias prehispánicas; v) potenciar y tal vez detonar procesos migratorios masivos, tanto entre áreas rurales como sobre todo campo-ciudad, con los consiguientes costos familiares y sociales y las demandas urbanas inherentes a estos procesos.

Las regiones contiguas VII, VIII, IX y X representan más de un quinto de la superficie nacional $(21,9 \%)$ y cerca de un tercio $(32,3 \%)$ de la población total (INE, 1992; MIDEPLAN, 1995). Con la excepción de la VIII Región del Bío-Bío, dichas regiones tienen una población rural cercana al $40 \%$ de su población total, mientras que en el país ese porcentaje alcanzaba sólo al $16,5 \%$ en el último censo. En consecuencia, las cuatro regiones concentran ni más ni menos que el $63,3 \%$ de la población rural nacional, casi dos tercios del total. Las provincias con mayor población rural son Linares $(37,0 \%$ del total rural de la VII Región); Ñuble (46,0\% de la VIII Región); Cautín. (75,0\% de la IX Región); y Valdivia (34,5\% de la X Región). (INE, 1992).

Económicamente, las cuatro regiones aportan algo menos de un quinto del producto nacional $(18,7 \%)$, contribuyendo, sin embargo, con el $43,3 \%$ del producto del sector silvoagropecuario. Este sector, que en 1990 representaba sólo el 9,0\% del PIB nacional, alcanzaba en esta regiones -exceptuada la VIII- valores del 20 al $30 \%$ de los correspondientes PIB regionales (Ministerio del Interior-CIEPLAN, 1994; MDEPLAN, 1995).

En relación a la fuerza de trabajo, la del sector agricultura, caza y pesca (no desagregado) ascendía, en 1994, a 436.000 personas en el conjunto de las cuatro regiones (con una distribución más o menos pareja entre ellas). Ese número representaba más de la mitad de la fuerza de trabajo sectorial nacional: un 55,9\%. La tasa de desocupación total (no sectorial, 1992) era mayor al promedio nacional en las regiones VII y VIII, y bastante inferior en las regiones IX y X (MIDEPLAN, 1995).

En exportaciones, la contribución de las cuatro regiones en 1994 representó cerca de un cuarto $(23,4 \%$ ) del total nacional. Este promedio incluye extremos correspondientes a la VIII Región, la tercera más exportadora del país, y a la IX, la menos exportadora, tanto en relación al total nacional como a su propio producto regional. La canasta exportadora de tres de las cuatro regiones -excepción hecha de la $\mathrm{X}$ - es predominantemente forestal y secundariamente agrícola y agroindustrial. Tales valores, agregados, explican entre el 65 y el $80 \%$ del valor exportado (aunque sólo cerca del 12\% en el caso de la X). Esta misma 
especialización se traduce en un fuerte aporte a las exportaciones sectoriales nacionales, aunque concentrado en las regiones VII y sobre todo VIII. En conjunto, las 4 regiones contribuyen con el 100\% de las exportaciones de pasta de madera y fibras celulósicas, el 93,6\% de las de madera y manufacturas de madera (el $33,7 \%$ de las de muebles y similares), el 51,1\% de las de preparados de legumbres y hortalizas, y sólo el 12,8\% de las de frutos comestibles (PROCHILE, 1993 y 1994).

El destino geográfico de las exportaciones regionales demuestra la escasa relevancia del MERCOSUR'para la mayoría de ellas. En efecto, con la excepción de la IX -con casi un tercio $(32,2 \%)$ de sus ventas a ese mercado- las otras tres regiones sólo destinan al MERCOSUR un 10,7\% (VII), 6,0\% (VIII), y 4,8\% (X) de sus exportaciones. (Prochile, 1993 y 1994).

En términos sociales, la pobreza afectaba, en 1994, a cerca de medio millón de personas entre la VII y X regiones, más de dos tercios $(68,6 \%)$ del total nacional rural. En cifras más desagregadas, el área interregional acumulaba el $71 \%$ de la indigencia rural del país, y el $67,8 \%$ de los pobres no indigentes. En uno y otro caso, las mayores concentraciones de pobreza, en términos absolutos, se radicaban también en la VII y VIII regiones, las mismas con mayores tasas de desocupación (MIDEPLAN, 1995).

El nivel de escolaridad -años de estudio, promedio-de la población económicamente activa era, en 1993, inferior al promedio nacional $(9,4$ años) en las cuatro regiones. Dos de ellas, la VII y la X, presentaban los menores niveles nacionales ( 7,7 y 7,8 años, respectivamente). Otro tanto sucede al analizar indicadores de salud tales como la esperanza de vida al nacer y la mortalidad general. Las cuatro regiones analizadas presentaban las menores esperanzas de vida de todo el país y las mayores tasas de mortalidad general (a excepción sólo de la Región de Valparaíso). (MiDEPLAN, 1995).

Así pues, el impacto negativo en el empleo-sectorial en estas regiones adquiere un singular dramatismo, agudizando los problemas estructurales de una agricultura tradicional vinculada a una pobreza rural extrema, y condicionando gravemente las opciones reales de una necesaria reconversión. 


\section{A. Daher / Tratados de Libre Comercio y mercados ...}

\section{Mercados regionales de trabajo y reconversión agraria.}

Los efectos de distinto signo de los TLC, y en particular del MERCOSUR, en la agricultura chilena implicarán una creación y reducción de empleos según los sectores beneficiados y perjudicados y las regiones ganadoras y perdedoras. La estructura según categorías de ocupación, diferenciada entre las "dos agriculturas" y sus respectivos territorios, acusará probablemente no sólo el cambio cuantitativo en los niveles de empleo, sino también la modificación cualitativa exigida por la inminencia de la reconversión de la agricultura tradicional.

Al desconocimiento de la real magnitud del impacto de los tratados en el empleo, y al consiguiente desconocimiento de los costos-económicos y sociales del ajuste y la reconversión, se suma una cierta duda fundada en relación a su propia viabilidad, vinculada a un conjunto de restricciones -naturales y sociales-que afectan la reasignación de los factores productivos -en especial tierra y trabajo-.

Territorialmente, los ajustes en los mercados regionales de trabajo -vía empleo, remuneración y cambio en las categorías ocupacionalesdetonarán probablemente la reasignación geográfica de este factor, con los consecuentes cambios demográficos.

\section{a) Impacto de los TLC en el empleo agrícola.}

Los diversos Acuerdos y Tratados de Libre Comercio implican efectos en el empleo sectorial de diferente magnitud y carácter.

Una primera evaluación -en el escenario de un ALC.con los Estados Unidos-indica que, para los productos agropecuarios importables, la disminución de la ocupación directa sería del orden del 10\% $(8.000$ personas); para los exportables, șe estima un incremento de 2.000 empleos, a los que se suman al menos otros 8.500 correspondientes a los exportables agroindustriales. Si bien el efecto neto es positivo para el país, el impacto no es neutro en términos interregionales. En efecto, la ocupación vinculada a los rubros importables cae en forma importante entre las regiones VII y IX (en las que predomina el cultivo de cereales, remolacha y oleaginosas). El mayor aumento asociado a los rubros exportables se localizaria en la VI Región, seguido por la V, la RM y la VII (donde se radica la producción de tomates para procesamiento, de duraznos para conservas y de pasas). El balance regionalizado favorece desde la IV a la VI regiones (centro-norte), resultando perjudicadas las regiones VII, VIII, IX y X (centro-sur y sur) (Muchnik et.al., 1992). 
Así pues, si bien un ACL con Estados Unidos tendría efectos casi neutros en el empleo sectorial agregado (Agüero, 1995) y otro tanto sucedería con el NAFTA, en este último caso las especificidades regionales son también importantes. Las potenciales ganancias favorecen a las regiones V y VI (zona central, con fruticultura, hortalizas y vitivinicultura de exportación), y a lá regiones IX y.X (sur; donde la ganadería aminoraría los efectos negativos de la contracción de cultivos de trigo y raps). El problema de empleo se concentraría, en consecuencia, con especial fuerza en las regiones VII y. VIII (centro-sur, con cultivos tradicionales), zona que además resulta ser la más afectada por la crisis actual de rentabilidad del sector (Quiroz, Larraín y Labán, 1995).

Los mismos autores concluyen que los efectos producidos por el NAFTA deben ser considerados como una aproximación "conservadora" de los efectos que se derivarian de la inserción de Chile en el MERCOSUR, los que impactarian nuevamente más a las regiones VII, VIII y IX. Complementariamente, debe tenerse presente sin embargo que se ha estimado (en relación a un ALC con Estados Unidos) un incremento en el empleo forestal de $9 \%$ al año 2000 (9.500 personas) (Morales y Fischer, 1992), el que se localizaria probablemente, en gran medida, en las mismas regiones.

En relación al MERCOSUR, como se ha advertido, no hay estudios especificos relativos al impacto laboral derivado de la asociación de Chile a ese mercado: A continuación se exponen, en primer lugar, algunas apreciaciones generales, y luego ciertas estimaciones hechas en esta investigación con el propósito de definir, preliminarmente, órdenes de magnitud.

Un partido político, Renovación Nacional, estimó en abril -antes de concluirse las negociaciones con MERCOSUR y de conocerse sus términos definitivos- que la asociación afectaría el empleo de 300 mil personas (El Mercurio, 07-04-1996). En similar momento, otra opinión identificaba un "impacto letal en 200 mil familias (un millón de personas) de agricultores minifundistas (...) y un retroceso importante en la calidad de vida de los 60 mil propietarios de parcelas de la Reforma Agraria, que transitarán hacia una agricultura de subsistencia" (Gazmuri, 1996: A2);

El Ministro de Economia, Alvaro García, sostuvo a su vez que el $80 \%$ de los trabajadores agrícolas se vería directamente beneficiado con el MERCOSUR (afirmando, junto a los Ministros de Relaciones Exteriores y Agricultura ante la Comisión Especial de la Cámara de Diputados, que MERCOSUR en su conjunto generaría $150 \mathrm{mil}$ nuevos empleos) ( $E l$ Mercurio, 25-07-1996, Al y 12). De aquí puede derivarse que cerca de 
160.000 ocupados agrícolas (20\% de un total de 814 mil en 1995) resultarian perjudicados $o$, en el mejor de los casos, no beneficiados por el MERCOSUR. (Al considerar sólo el porcentaje del empleo "directo" en el subsector "cultivos" (Carrasco y Véliz, 1996) tal cifra bajaría a 15.200 empleos).

En términos de empleo, la disminución de hectáreas señalada -en el anterior capítulo- para cada cultivo permite concluir que, dadas las jornadas-hombre por hectáreas requeridas en promedio por cada uno de ellos (Anuario del Campo, 1992-1993), la reducción total alcanzaría a 2,9 millones de jomadas-hombre, correspondiendo tres cuartas partes a remolacha -altamente intensivo en trabajo-, un $14,4 \%$ a trigo, $5 \%$ a maiz y el resto a oleaginosas y arroz. (Esto equivale a alrededor de 10.500 empleos).

Estas cifras se comparan con la "aproximación conservadora" de Quiroz, Larraín y Labán (1995) que señala una reducción de 15.000 empleos concentrada principalmente en las regiones VII, VIII y IX. En otro escenario, que excluye el libre comercio de trigo pero con fuerte incidencia de las pérdidas de empleo en remolacha, los mismos autores calculan una reducción de casi 50.000 empleos.

Con un método distinto -considerando la relación sectorial agrícola entre personas ocupadas y hectáreas cultivadas-se puede determinar que la pérdida de 106 mil hectáreas de cultivo (Muchnik, Errázuriz y Domínguez, 1996) involucraría a 50:476 empleos (atendiendo que, en promedio, cada persona ocupada cultiva 2,1 hectáreas) (Carrasco y Véliz, 1996). Esta cifra subiría a 71.428 empleos si, según afirman Hachette y Morales (1996), las menores hectáreas cultivadas serían 150 mil; apro-ximadamente.

Otro procedimiento de cálculo, basado en la relación entre valor exportado y empleo -200 personas por cada millón de dólares en el sector agropecuario en 1991, según Meller y Repetto (1996)-permite determinar una reducción de 53.160 empleos, atendiendo la pérdida ya señalada de US\$265,8 millones. (Debe considerarse, sin embargo, que las agriculturas tradicional y exportadora pueden diferir en la intensidad de mano de obra).

Así pues, es claro que el impacto laboral no genera consenso entre los gremios, los partidos políticos, el gobierno ni, incluso, entre los académicos. Este es un tema ciertamente no cerrado y de la mayor relevancia económica y social.

Si bien, como se ha señalado, algunas de las pérdidas de empleo en el subsector de cultivos podrían compensarse por la creación de empleos 
en otros subsectores silvoagropecuarios "ganadores" con el MERCOSUR, y también con el presunto mayor empleo en otros sectores de la economía -como industria y servicios-, no es menos cierto que, incluso en tales casos, tal ajuste supone un costo económico y social e incluso flujos migratorios y reordenamientos demográficos.

Debe tenerse presente que, proporcionalmente, el empleo en el sector cultivos representa casi un tercio (32\%) del empleo sectorial agrícola, y el de cereales, por sí solo, un $19 \%$. En contraste, el sector frutícola equivale a un $21 \%$. Las transferencias y reasignaciones del factor trabajo al interior del sector agrícola presentan, pues, obvias restricciones. Es necesario considerar además que no sólo los menores empleos sino también los menores precios pueden generar, en el largo plazo, flujos migratorios de trabajo y capital fuera del propio sector agrícola (Quiroz, Larraín y Labán, 1995). La población rural podría bajar del 17\% actual al $10 \%$ de la población total en quince años más (Eduardo Salgado, en Revista del Campo, 1996).

Ahora bien, además de preevaluar los impactos cuantitativos en el empleo sectorial es relevante intentar prever cambios cualitativos en el mismo asociados al libre comercio agropecuario y a la reconversión y modernización inducida por él.

En términos de mercados de trabajo, es interesante observar, como antecedente, su estructura según categoría de ocupación y las variaciones registradas en la misma en el período postrecesivo 1986-94. Mientras el número de ocupados prácticamente no varía, el de empleadores acusa una disminución de $5,1 \%$ y los ocupados por cuenta propia muestran un importante incremento cercano al $41 \%$. Los asalariados, a su vez, presentan un número más bien constante, el cual sin embargo registra fuertes variaciones al desagregar esta categoria según sexo: los hombres decrecen en $19,3 \%$ mientras las mujeres asalariadas suben en $21,7 \%$ (Carrasco y Véliz, 1996).

Esta estructura según categoria de ocupación -que ya alude a mercados laborales diferenciados, aunque con cierta movilidad entre ellospresenta, además, un interesante correlato regional. En efecto, las regiones más campesinas, con mayor proporción de trabajadores por cuenta propia, son -además de las del Norte Chico, poco significativas en términos absolutos-las que se ubican entre el Maule y Chiloé (VII a X). En cambio, aquellas más acentuadamente empresariales, con preponderancia de asalariados, son las localizadas entre Aconcagua y Maule (regiones V, Metropolitana y VI), en el centro del país (Gómez y Echenique, 1988). 
Así pues, la superposición de distintas estructuras de categorías ocupacionales con territorios específicos y, además, con subsectores agrícolas diferenciados en esas mismas regiones determina que, en el caso chileno, los mercados regionales de trabajo tengan, simultáneamente, una especificidad socioeconómica y sectorial, circunscrita en verdaderas "áreas de mercado".

Estas "áreas" de mercado constituirían en consecuencia no sólo barreras geográficas a la movilidad laboral, sino además ámbitos "naturales" de capacitación práctica especifica del factor trabajo, haciéndolo más "especializado" o menos versátil. Las mismas áreás definen asimismo relaciones de producción particulares, constitüyéndose en ámbitos socioculturales más o menos singulares.

Mención especial, en relación a la estacionalidad y movilidad geográfica, debe hacerse de la fuerza de trabajo temporal -unas $400 \mathrm{mil}$ personas según la SNA, Sociedad Nacional de Agricultura-. Esta sumaba, en el periodo $1964-65,147$ mil personas; 198 mil entre 1975-76; y 300 mil en 1986-87. En la década de los ochenta-donde se registra el auge exportador agrícola y en especial frutícola- entre un 20 y un $30 \%$ del contingente total de la fuerza de trabajo agrícola era de origen urbano. Este porcentaje llegaba al $50 \%$ en la zona central durante los meses de cosecha en la fruticultura. Un 13\% de los temporeros residía habitualmente en grandes ciudades, y un $3,1 \%$ de los mismos lo hacía en Valparaíso y Santiago (Gómez y Echenique, 1988).

Este fenómeno de reversión relativa de la tradicional migración campo-ciudad no se expresaba sólo en la urbanización de una cierta proporción de la fuerza de trabajo agricola, sino también en la ruptura de la antigua "dualidad" rural-urbana manifiesta en la modernización de la agricultura -en especial la de exportación-e incluso en la integración intersectorial e interregional de las empresas, tanto productivas como de distribución y prestación de servicios en general. Se prefiguraba así, a fines de los ochenta, una "agrourbanización" orientada a la exportación (Daher, 1987).

El mercado laboral itinerante de los "temporeros" -siguiendo los desplazamientos geográficos de la estacionalidad vinculados a la gran variación latitudinal de la agricultura chilena-sugiere un encadenamiento, en términos de mercados regionales de" trabajo, entre las "dos agriculturas", donde ciertamente una, la tradicional, es tributaria de la otra.

Estos temas, como también el de los probables cambios cualitativos en la estructura por categorías de ocupación, requieren por cierto de un estudio específico que excede los alcances de este primer documento. 
Ellos resultan por de pronto muy sugerentes en relación a la evolución de los mercados regionales de trabajo en el escenario de la reconversión de la agricultura tradicional chilena.

\section{b) Opciones y restricciones en la reconversión.}

La reasignación sectorial y territorial de los factores productivos -en especial tierra y trabajo-, en el contexto de una reconversión de la agricultura tradicional chilena, presenta opciones y restricciones apenas esbozadas al momento de suscribirse la asociación de Chile al MERCOSUR.

En el corto plazo, la baja de precios reasignará el factor tierra entre cultivos y actividades agrícolas, con una elasticidad relativamente reducida. A mayor plazo, aquella puede generar flujos migratorios de trabajo y de capital entre la agricultura y el resto de la economía, induciendo así respuestas mayores a los cambios de precios (Quiroz, Larrain y.Labán, 1995).

En relación a la tierra, su reconversión implicaría territorios a la vez extensos en superficie y especificos en localización. Si se considera que el 75\% de trigo se concentra en las regiones VII, VIII y IX (mayoritariamente en zonas de secano); que el $64 \%$ del maíz se produce en la VI Región (porcentaje que sube al $93 \%$ al incluir la Región Metropolitana y la VII); que el 100\% de la superficie arrocera está en las regiones VI y VII y en la provincia de Ñuble; que más de la mitad de las siembras de raps se concentran en la IX Región, y las de maravilla entre la Metropolitana y la VIII, aunque sobre todo en la VII; y que el cultivo de remolacha se extiende entre las regiones VI y X (Véliz, 1995), entonces es posible prever cambios en el uso de la tierra -con todo lo que esto implica-en áreas del país históricamente organizadas en función de la agricultura tradicional y de cultivos anuales que, en 1993-1994, comprometían a más de 700.000 hectáreas.

La reconversión de tierras está sujeta, sin embargo, a algunas consideraciones relativas a elasticidad, sustituibilidad y complementariedad. En efecto, la elasticidad de la superficie de trigo en relación a su propio precio es 0,85 , lo que implica que una baja de $10 \%$ en el precio reduce en $8,5 \%$ (35.000 hectáreas) su superficie. A su vez, un menor precio del trigo induce sustitución hacia remolacha -con una elasticidad cruzada alta de $-2,51-$ y ganado de vacuno (una reducción de $10 \%$ en el precio del trigo induce un aumento de $1,4 \%$ en la masa ganadera). Adicionalmente, por complementariedad, las estimaciones econométricas indican 
que esa misma baja en el precio del trigo genera una reducción de 14,6\% en la superficie de raps ( $y$, dada la elasticidad-precio de la superficie de trigo, una relación casi uno a uno entre las superficies de ambos). Los rubros sin relaciones de sustituibilidad ni complementariedad fuertes, y por ende más independientes, son maíz, arroz y maravilla, con elasticidades-precio de $0,4,0,4$ y 2,6 , respectivamente. Pues bien, en cualquiera de los dos acuerdos, NAFTA o MERCOSUR, se estima una caida en la superficie de trigo de $22 \%$ como promedio, con efecto indirecto -por el patrón de rotación-en las oleaginosas, las que, al levantarse las bandas como efecto del MERCOSUR, resultan eliminadas. Así pues, una baja de precios adicional a los problemas de competitividad estructural de la agricultura produciría un cambio radical en la agricultura nacional, impactando el precio relativo de la tierra y con ello la estructura productiva completa, orientándola probablemente hacia mayores escalas de explotación, métodos de producción más mecanizados y estrategias más extensivas (Quiroz, Larrain y Labán, 1995).

Existen por cierto otras restricciones a la reconversión de tierras tanto o más importantes. De hecho, a niveles de región o provincia, y sobre todo a nivel individual involucrando a miles de productores, persisten situaciones que incapacitan la reorientación de sus rubros tradicionales. Factores relacionados con limitaciones de recursos naturales (tipo de suelo y clima), localización geográfica, capacidad económica y empresarial, además de dificultades de acceso al crédito, a la mecanización, a la asistencia técnica y a la información (Véliz, 1995), condicionan o inhiben los procesos de modemización y reconversión.

La reconversión de la agricultura tradicional chilena enfrentará dificultades asociadas a la fragmentación de la propiedad -subdivisión predial-que puede imposibilitar o complicar la obtención de economías de escala necesarias a la competitividad internacional propia de una economía abierta e integrada; a la disimil calidad de suelos y opciones de reconversión en el uso de la tierra, complejizadas por las fluctuaciones climáticas propias de una realidad geográfica de amplia de variación latitudinal; y a la desigual disponibilidad y acceso a infraestructuras de transporte (en especial viales, ferroviarias y portuarias), de riego, $y$, en menor medida, energéticas y de comunicacionès.

En términos de capital financiero, la reconversión deberá enfrentar la muy heterogénea capacidad de los pequeños y medianos propietarios y empresarios agrícolas para acceder a los mercados financieros y a créditos compatibles con los plazos y riesgos de la reconversión, y competitivos desde la perspectiva de los costos involucrados. Ello se ve 
agravado por las condiciones de pobreza y extrema pobreza, la existencia de propiedad comunitaria, la irregularidad de títulos de tenencia, etc.

Pero tal vez una de las mayores dificultades es la reconversión del capital humano propiamente tal. Gran parte de la fuerza de trabajo y del empresariado rural de estas regiones están condicionados por las características deficitarias de educación, salud, vivienda y, en generai, por una situación social de pobreza e indigencia. Ello se agrava por los mayores costos de proveer infraestructura y servicios sociales dada la dispersión geográfica de la población rural. La existencia o insuficiencia de estos últimos hipoteca la opción de cambio estructural, al menos en el cortomediano plazo, dejando inhabilitada a buena parte de la población frente a los desafios de la reconversión.

Todo lo anterior hacía recomendable preparar el ajuste agricola para reducir su costo, incentivando la movilidad de los recursos dentro del agro (a la silvicultura, particularmente) y hacia fuera de ese sector, e incentivando la reconversión rápida de tierras cuya producción tradicional se vería afectada por la mayor apertura al exterior, cualquiera sea la forma adoptada en su consecución (Hachette y Larrain, 1994): Obviamente la mayor movilidad intra e intersectorial de recursos y la reconversión de tierra determinarán cambios de significación en los territorios rurales, e incluso urbanos, modificando la geografia económica y social de varias regiones del país.

En relación a lo anterior la participación de organizaciones gremiales, sobre todo empresariales, en el desarrollo de las negociaciones de la asociación de Chile al MERCOSUR contrasta con la menor o nula participación de los gobiernos regionales, en especial de las regiones "perdedoras", en los términos acordados en las mismas. A su vez, la voz de los partidos y conglomerados políticos se ha hecho oír mucho más que la opinión, prácticamente ausente, de las organizaciones de trabajadores y de los sectores laborales que podrian resultar más directamente afectados.

En medio de la contingencia y de la polémica pública no han faltado propuestas de extrema radicalidad, que plantean: ayudar a los empresarios agricolas a invertir en los países del MERCOSUR, ya que "si no pueden crecer como empresarios aquí, que lo hagan allá"; educar a las generaciones jóvenes de campesinos "para que puedan salir del sector en forma exitosa"; reemplazar líneas de crédito que financiarian pérdidas irrecuperables "por líneas para concentrar la propiedad"; y, en vez de subsidiar cultivos y bandas de precios, subsidiar a las industrias, colegios y universidades que se muevan a las regiones rurales (Barros, 1996). 
Por su parte, la Sociedad Nacional de Agricultura formuló -en Temuco- un conjunto de medidas o requerimientos para la modernización del sector y la minimización de los efectos negativos del MERCOSUR. El gobierno, a su vez, a través del Ministerio de Agricultura, acogió favorablemente algunas de dichas demandas, como el aumento de las inversiones en regadío, la renovación de la vigencia del subsidio a la forestación, la disponibilidad de créditos en dólares con tasas preferenciales, la bonificación de inversiones y la revisión institucional y funcional de la Comisión de Distorsiones. En cambio, en relación a la demanda de supresión de aranceles para la importación de insumos y bienes de capital del sector, el gobiemo no variaría la estructura aduanera común para el conjunto de la economía, cuidando sin embargo la inclusión de aquellos en las listas de desgravación inmediata en las negociaciones comerciales con paises y bloques supranacionales (El Mercurio, 11-07-1996: B1; 22-08-1996: B1 y B5).

Estas medidas se sumarian a algunas más específicas implementadas en atención a problemas estructurales del agro, que el MERCOSUR sólo haría más evidentes y agudos, tales cömo el programa de reconversión de áreas críticas -arroceras y viticultura en zonas de secano-y los proyectos de desarrollo campesino, de crédito para campesinos y mapuches pobres de La Araucanía, y de desarrollo para comunas pobres del área de secano, entre otros (Ministerio de Agricultura, 1996 a).

Si bien las medidas gubernamentales reseñadas más atrás tendrían, indirectamente, incidencia en el empleo sectorial, no es menos cierto que la ausencia de un pronunciamiento explícito sobre el mismo y sus consecuencias sociales y demográficas sólo puede entenderse a partir del desconocimiento generalizado de la real magnitud del impacto laboral agrícola, en especial en mercados regionales especificos del factor trabajo.

$\mathrm{Al}$ respecto, y con anterioridad a la respuesta gubernamental comentada, el Ministro de Agricultura habría planteado, en términos generales, dos escenarios posibles para la agricultura chilena del futuro: uno, donde ella, "generadora de empleo rural bien remunerado", se orientaria a productos de alta calidad y valor unitario, basada en èl desarrollo de los mercados externos y en la transformación técnica, productiva y comercial. Otro, correspondiente a una agricultura que, aprovechando igualmente las tendencias del mercado internacional privilegiaría la inducción de fuertes inversiones de capitales, mayores escalas de producción y más bien extensivas, particularmente de ganadería y actividades silvícolas, "con menorincidencia en el empleo". Ante estas alternativas, el Ministro 
plantea como la opción más realista una combinación de ambas (Ministerio de Agricultura, 1996 b).

¿Políticas o medidas más especificas para las diversas posibilidades de reconversión agraria según calidades de suelo, condiciones climáticas, fraccionamiento predial, disponibilidad de infraestructura? ¿Medidas apropiadas a la cantidad y calidad de los recursos humanos involucrados, a sus niveles de escolaridad y capacitación, a sus tradiciones comunitarias? ¿Políticas regionales y locales orientadas a las áreas territoriales mayormente impactadas con el MERCOSUR, con problemas estructurales propios y anteriores a este último, y con marcadas especificidades subsectoriales -por rubros o cultivos-, concentrados muchas veces en provincia e incluso comunas particuläres?

\section{Conclusiones.}

Las principales conclusiones de este estudio se presentan a continuación refiriéndolas a: a) los Tratados de Libre Comercio y los mercados regionales de trabajo; b) sus efectos en la agricultura y en las regiones de Chile; y c) sus efectos en el empleo y en las opciones de reconversión agricola.

\section{a) Tratados de Libre Comercio y mercados regionales de trabajo:}

1. El libre comercio se basa en y afecta a los mercados de factores, incluido el del trabajo, tanto è el ámbito intemacional como subnacional, y según las especificidades sectoriales y territoriales existentes no sólo entre países, sino también entre regiones.

2. Los Tratados de Libre Comercio, aún beneficiando al país en su conjunto, pueden favorecer más o menos a determinados sectores y regiones, y perjudicar a otros. Los balances agregados de empleo pueden ocultar, en consecuencia, desbalances sectoriales y regionales en los mercados de trabajo.

3. Los mercados regionales de trabajo -que acusan diferencias en la evolución de la fuerza de trabajo, la cesantía y los niveles de salario-no sólo están territorialmente definidos, sino también lo están -en el caso de la agricultura chilena- por una especialización subsectorial, y por relaciones laborales y productivas singulares, dentro de un ámbito socioeconómico y cultural propio. 


\section{b) TLC, efectos en la agricultura y en las regiones de Chile:}

1. El libre comercio afectará con signo contrario a las "dos agriculturas": la modema, empresarial y exportadora se verá beneficiada; mientras que la tradicional, campesina y sustitutiva, resultará en general perjudicada. La primera, geográficamente central, hara ganadoras a las regiones V, VI y Metropolitana; la segunda, propia del centro-sur y sur del país, determinará que las regiones VII, VIII, IX y X resulten perdedoras.

2. Estas cuatro regiones concentrarán dos tercios de las pérdidas sectoriales, regionalizadas en proporción a la distribución de la superficie cultivada pertinente. Al corregir dicha asignación según los diferenciales geográficos de rendimientos, las pérdidas inicialmente decrecientes hacia el sur, se acentúan precisamente en esa misma dirección.

3. El impacto sectorial negativo involucra a cuatro regiones contiguas, afectando a pequeños y medianos propietarios y empresarios, a comunidades indígenas y a sectores de pobreza e indigencia, amenazando formas de vida y culturas arraigadas en la ruralidad, y potenciando migraciones masivas con alto costo económico y social y fuerte impacto urbano.

4. Las cuatro regiones perdedoras representan un quinto de la superficie nacional y un tercio de la población total. De esta, algo más del 16\% es rural en el pais, mientras que en estas regiones -con excepción de la VIII-dicho porcentaje alcanza al $40 \%$. Ellas contribuyen con el $43 \%$ del producto silvoagropecuario, sector que representa entre el 20 y el $30 \%$ del PIB de las mismas, y sólo el 9\% del PIB nacional.

5. El impacto negativo de MERCOSUR en el empleo agrícola adquiere connotaciones territoriales muy graves si se considera que las cuatro regiones perdedoras concentran más de la mitad de la fuerza de trabajo sectorial (436 mil personas), más de dos tercios de la pobreza nural del país (medio millón de personas) y tres cuartas partes de las indigencia nacional no urbana, con los peores índices de educación y salud.

\section{c) TLC, efectos en el empleo y en la reconversión agrícola:}

1. La carencia de estudios especificos sobre el impacto laboral de los TLC, y en especial del MERCOSUR, redunda en un desconocimiento de la real magnitud del mismo. Ello impide, a su vez, conocer un componente importante de los costos -económicos y también sociales-del ajuste y la reconversión sectorial. 
2. Estimaciones más bien conservadoras permiten asociar a la reducción de 100 a 150 hectáreas cultivadas una pérdida de 50 a 7.0 mil empleos directos, como efecto del MERCOSUR. Debe tenerse presente que este orden de magnitud se da en un contexto donde el empleo del subsector cultivos representa un tercio del empleo sectorial agrícola, y el de cereales, por sí solo, casi un $20 \%$, levemente inferior al frutícola.

3. Además de los cambios cuantitativos en el empleo, MERCOSUR inducirá modificaciones cualitativas en la estructura por categorias ocupacionales, sustituyendo aquella más propia de una agricultura tradicional campesina por otra pertinente a una agricultura moderna empresarial.

4. En efecto, en el conjunto de la estructura nacional agrícola según categorias de ocupación se observa, en el período 1986-94 y para un número absoluto de empleo prácticamente constante, un incremento significativo superior al $40 \%$ de ocupados por cuenta propia y, para un número de asalariados también constante, un decremento de $20 \%$ en la participación masculina compensado por un incremento similar en la femenina. MERCOSUR sin duda detonará cambios incluso más significativos y geográficamente más concentrados.

5. Los mercados regionales de trabajo, en el sector agrícola chileno, se revelan así caracterizados y définidos múltiplemente por su dimensión geográfica local, su especialización subsectorial, su propia estructura según categorias de ocupación y, asociadas a ella, sus relaciones laborales propias en medio de un ámbito social y cultural específico.

6. La reconversión de la agricultura tradicional involucrará cieitamente una reconversión en los mercados de factores en generál y de trabajo en particular. Debe considerarse que no sólo ló menöres empleos, sino también los menores precios y salarios pueden iǹducirflujos migratorios de capital y trabajo dentro y fuera del sector agrícola y de las regiones afectadas.

7. La reconversión de la agricultura tradicional, acelerada como consecuencia del MERCOSUR, enfrentará sin embargo importantes restricciones relacionadas con limitantes de recursos naturales, en especial calidades de suelos y climas según localización geográfica; elasticidades, sustituibilidades y complementariedades en el uso de la tierra y en sus ciclos de rotación; fragmentación predial e irregularidades en los títulos de tenencia, dificultando la obtención de economias de escala y el acceso al crédito; desigual dotación de infraestructura, en especial de riego y transporte; capacitación empresarial y laboral y modernización tecnológica inhibidas por las condiciones de pobreza e indigencia y los bajos indices de escolaridad y salud. 
8. La fuerte incidencia directa e indirecta de la agricultura en el PIB de las regiones afectadas, su gran porcentaje de población rural, las condiciones extremas de calidad de vida de una gran proporción de la misma, y el impacto de cesantía, mayor pobreza y migraciones esperado, plantean la urgencia de formular, además de medidas sectoriales específicas, políticas regionales y locales que corrijan el caso omiso que las recientes estrategias regionales de desarrollo hacen del MERCOSUR.

\section{Referencias bibliográficas.}

Anuario del Campo, "Cultivo por cultivo", Anuario del Campo 1992-93. Santiago: Publicaciones Lo Castillo, 1992-1993, Capitulo IV; pp. $112-$ 159.

Barros, César, "Sector agropecuario en Chile. Un desafío para tigres", Puntos de Referencia, $N^{\circ} 168$, Centro de Estudios Públicos, Santiago, 1996.

Carrasco, Eduardo y Véliz, Héctor, "El mercado de trabajo del sector agrícola 1986- 1995 ", Serie Documentos de Trabajo, No244, Centro de Estudios Públicos, Santiago, 1996.

Daher, Antonio, "Agrourbanización 'for export"', EURE, Santiago, Vol. XIV, 1987, №1, pp. 7-14.

Daher, Antonio, "Competencia, regiones ganadoras y perdedoras en Chile", EURE, Santiago, 1994, Vol. XX, No60, pp. 63-84.

Daher, Antonio, "Las regiones de Chile frente al NAFTA y al Mercosur", Serie Documentos de Trabajo, № 249, Centro de Estudios Públicos, 1996 a; y EURE, Vol. XXII, N’66, Santiago, 1996 a.

Daher, Antonio, "Tratados y exportaciones regionales de Chile", Estudios Públicos, N63, Centro de Estudios Públicos, Santiago, 1996 b.

El Mercurio, "RN propone «Plan Pro Agro»", Santiago, 7 de abril de 1996, p. C3.

El Mercurio, "Ocho medidas paliativas solicitan agricultores al gobierno por Mercosur", Santiago, 11 de julio de 1996, p. B1.

El Mercurio, "Esfuerzos para aclarar efectos del Mercosur", Santiago, 25 de julio de 1996, p. A1 y A12.

ElMercurio, "Balance favorable en la Cámara para aprobación del Mercosur", Santiago, 27 de julio de 1996, p. C4.

El Mercurio, "Gobierno fijó su política para compensar al agro", Santiago, 22 de agosto de 1996, p. B1 y B5.

Errázuriz, Luis Felipe y Muchnik, Eugenia, "Visión crítica de la agricultura chilena y sus políticas", Serie Documentos de Trabajo, No245, Centro de Estudios Públicos, Santiago, 1996. 
Escobar, Bemardita y Meller, Patricio, "Efecto regional del modelo exportador: evolución del diferenciali salarial entre regiones y Santiago", enP. Meller (ed.), El modelo exportador chileno, crecimiento y equidad. Santiago: CIEPLAN, 1996, pp. 141-188.

Escobar, Bernardita y Repetto, Andrea, "Efectos de la estrategia de desarrollo chilena' en lầs regiones: una 'estimación' de la 'rentabilidad del sector transable regional"; 'Colención Estudios CIEPLAN, N37, Santiago, 1993, pp. 5-36.

Faiguenbaum, Hugo, "Cultivos amuales: estadísticas sobre superficies, rendimientos, producciones, exportaciones e importaciones". Publicación docente, Santiago, 1996.

Ferrer, Aldo, "Mercosur: trayectoria, situación actual y perspectivas", Desarrollo Económico, Vol. 35, N 140, Buenos Aires, 1996, pp. 563-583.

Gazmuri, Renato, "Futuro de la agricultura", El 'Mércurio, Santiago, 24 de marzo de 1996, p. A2.

Gómę, Sergio y Echenique, Jorge, La agricultura chilena, las dos caras de la modernización. Santiago. FLACSO-AGRARIA, 1988.

Hachette, Dominique y Larrain, Felipe, "Una estrategia de inserción comercial para Chile", en: F. Larrain (ed.), Chile hacia el 2000. Ideas para el desarrollo. Santiago: Centro de Estudios Públicos, 1994, pp. 289-322.

Hachette, Dominique y Morales, Gustavo, "Impactos regionales de NAFTA y Mercosur", Serie Documentos de Trabajo, No254, Centro de Estudios Públicos, Santiago, 1996.

INE, "Censo de Población y Vivienda. Chile, 1992. Resultados Generales". Instituto Nacional de Estadísticas, Santiago, 1992.

Meller, Patricio y Repetto, Andrea, "Empleo y remuneraciones en el sector exportador chileno", en P. Meller (ed.), op. cit., pp. 189-212.

Meller, Patricio y Sáez, Raúl, "Lecciones y desafios futuros del auge exportador chileno", en: P. Meller, y R. Sáez (eds.), Auge exportador chileno, lecciones $y$ desafiós futuros. Santiago: CIEPLAN /DOLMEN, 1995, pp. 1-42.

Meller, Patricio y Tokman, Andrea, "Apertura comercial y diferencial salarial en Chile", en: P. Meller, op. cit, pp. 87-140.

MIDEPLAN, "Compendio Estadisticas Regionales", Ministerio de Planificación y Coordinación, Santiago, 1995.

Ministerio de Agricultura, "Balance de gestión 1994-1995. El desafio del desarrollo agro-rural", Ministerio de Agricultura, Santiago, 1996 a.

Ministerio de Agricultura, "El escenario de la agricultura chilena hacia el año $2010^{n}$, Informativo de Nuestra Tierra, año 1, N6, publicación mensual del Ministëno de Agricultura, Santiago, 1996 b, p. 2. 
Ministerio del Interior-CIEPLAN, "Evolución del producto por regiones, 19601992", Ministerio del Interior, Subsecretaria de Desarrollo Regional y Administrativo, Santiago, 1994.

Muchnik, Eugenia, et.al., "Efectos esperados de un acuerdo de libre comercio entre Chile y EE.UU. en los sectores agropecuario y agroindustrial chilenos", Confederación de la Producción y el Comercio, Santiago, 1992.

Muchnik, Eugenia; Errázuriz, Luis Felipe y Dominguez, Juan Ignacio, "Impacto de la asociación de Chile al Mercosur en el sector agricola y agroindustrial", Serie Documentos de Trabajo, N253, Centro de Estudios Públicos, Santiago, 1996.

Ortiz, Luis, "Un análisis regional del mercado de trabajo en México", Economía Informa, No245, UNAM, México, 1996, pp. 27-32.

Prochile, "Estadisticas de exportaciones regionales", Ministerio de Relaciones Exteriores, Dirección de Promoción de Exportaciones, Santiago, 1993 y 1994.

Quiroz, Jorge; Larrain, Felipe y Labán, Raúl, "El sector agrícolay agroindustrial frente a NAFTA y Mercosur". Trabajo preparado para la Sociedad Nacional de Agricultura, Santiago, 1995.

Revista del Campo, "Mejor rentabilidad vía reducción de costos", Revista del Campo, Santiago, N1044, p. A15.

Tironi, Emesto, La Organización Mundial de Comercio y la Konda Uruguay. Santiago: DOLMEN, 1995.

Véliz, Héctor, "El subsector de cultivos anuales y el NAFTA", Serie Documentos de Trabajo, N2230, Centro de Estudios Públicos, Santiago, 1995. 